Original Article

\title{
A CORRELATIVE STUDY ON DETERMINANTS OF TIME DOM AIN MEASURES OF CARDIAC VAGAL FUNCTION IN HEALTHY SUBJECTS
}

\author{
Subbalakshmi N.K. ${ }^{1}$, Jeganathan P.S. ${ }^{2} \&$ Sathyanarayana Rao K.N. ${ }^{3}$ \\ ${ }^{1}$ Associate Professor, ${ }^{2}$ Professor, Department of Physiology, Kasturba Medical College, Manipal University, \\ ${ }^{3}$ Professor, Department of Pharmacology, K.S. Hegde M edical Academy, Nitte University, \\ M angalore, Karnataka, INDIA. \\ Correspondence: \\ Subbalakshmi N.K. \\ Department of Physiology, Kasturba M edical college, M anipal University, Light House Hill Road, M anglaore - 575001 , \\ Karnataka, India. M obile: +91 94839 10879, Fax: (91) 8242428183 Email: rao.subbalakshmink@ rediffmail.com
}

\begin{abstract}
:
Introduction: E: I ratio and r-M SSD are known indicators of cardiac vagal activity. But their determinants are unclear.

Objective: To evaluate the determinants of E:I ratio and r-M SSD in healthy subjects.

M aterials and Methods: 141 healthy subjects were studied. In them body mass index (BMI), Q-T interval, heart rate (HR), systolic, diastolic, pulse and mean arterial blood pressure (M ABP) were measured. Age and sex of the subjects were noted. E: I ratio and r-M SSD was estimated from one minute lead II electrocardiogram in supine position. E: I ratio was recorded while breathing at 6 respiratory cycles per minute and r-MSSD was quantified during normal breathing. Data was analyzed by Pearson Correlation Coefficient and unpaired t test. $p$ value less than 0.05 was considered significant.
\end{abstract}

Results: There was positive correlation between E: I ratio and r-M SSD $(p=0.006)$. E: I ratio and r-M SSD were negatively correlating with age $(p<0.01)$. r-M SSD was negatively correlating with HR, systolic, diastolic, pulse and M ABP $(p=0.0001,0.01,0.03,0.04,0.01$ respectively). r-M SSD did not correlate with BMI and Q-T interval. E: I ratio did not correlate with blood pressure, BMI and Q-T interval. M ean E: I ratio and r-M SSD of males did not differ compared to females.

Conclusion: E: I ratio and r-M SSD are correlated. Age is an influencing factor of E: I ratio and r-MSSD. Blood pressure and HR are the other determinants of r-M SSD but not of E: I ratio in healthy subjects.

Key words: E: I ratio, r-M SSD, blood pressure, heart rate, vagal function, healthy subjects

\section{Introduction:}

The autonomic nervous system plays an important role not only in physiological situations, but also in various pathological settings such as diabetic neuropathy (1and 2), myocardial infarction ${ }^{(3 \text { and } 4)}$ and congestive heart failure. ${ }^{(5 \text { and }}$

${ }^{6)}$ Autonomic imbalance associating increased sympathetic activity and reduced vagal tone has been strongly implicated in the pathophysiology of arrhythmogenesis

\begin{tabular}{|c|}
\hline Access this article online \\
\hline Quick Response Code \\
\hline \\
\hline
\end{tabular}
and sudden cardiac death. $(7$ and 8) The beat of the healthy heart is not absolutely regular. This fluctuation in heart beat is known as heart rate variability (HRV). The ability of HRV analysis to assess overall cardiac health and the state of the autonomic nervous system responsible for regulating cardiac activity underlies its promise as a major new tool in the diagnostic and monitoring armamentarium. ${ }^{(9)}$ In the intact heart parasympathetic fibers are inhibitory and sympathetic are excitatory. Inhibitory actions of cardiac parasympathetic nerves are reported to provide electrical stability to the heart and thus preventing ventricular tachycardia in humans. ${ }^{(10)}$ Vagal nerve traffic cannot be measured directly in humans. The assessment of HRV has thus become the most widely-used indirect measure of cardiac vagal function.

Numerous methods of analyzing the magnitude of sinus

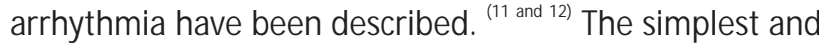
widely performed measure is the $\mathrm{E}$ : I ratio - a ratio of the 
longest $\mathrm{R}-\mathrm{R}$ interval in expiration to the shortest $\mathrm{R}-\mathrm{R}$ interval in inspiration during deep breathing. E: I ratio is a widely used indicator of parasympathetic function. ${ }^{(13)} \mathrm{r}$ MSSD is another time domain measur of HRV which is usually obtained during spontaneous breathing. r-M SSD reflects the short-term variation in the R-R intervals as they are entirely based on comparisons between successive beats. r-M SSD reflects alterations in autonomic tone that are predominantly vagally mediated and regarded as a reliable index of cardiac parasympathetic activity. ${ }^{(14 \text { and 15) }}$

A recent study exploring the possible clinical utility of time domain measures of HRV in monitoring cardiac autonomic function in type 2 diabetics found that, E:I ratio was significantly lower in type 2 diabetics compared to nondiabetic healthy subjects; but r-M SSD another indicator of cardiac parasympathetic function was comparable between type 2 diabetics and non-diabetic healthy subjects. In this study, in diabetics with cardiovascular complication r-MSSD declined significantly in one year period but E:I ratio did not decline significantly. However, in diabetics with somatic neuropathy both r-M SSD and E: I ratio declined in one year period. ${ }^{(16)}$ This observation prompt us to hypothesize that although r-M SSD and E:I ratio quantify cardiac parasympathetic function, the components of cardiac parasympathetic function quantified by these two HRV parameters may not be the same. Thus even though the analysis of HRV has gained popularity as a simple and non-invasive tool for assessing autonomic function in both normal subjects and in patients in a variety of clinical settings all the influencing factors of these HRV parameters remains uncertain. Consequently, we consider that analyzing influencing factors of HRV in different set of population may provide better insight in to making HRV a valuable tool in assessing autonomic function in different physiological or pathological conditions. Thus this study was undertaken to compare the association of E:I ratio and r-M SSD with age, gender, body mass index, systolic blood pressure, diastolic blood pressure, pulse pressure, mean arterial blood pressure, heart rate and QTc in healthy subjects. This study also sort correlation between E:I ratio and r-MSSD in healthy subjects.

\section{Materials and methods:}

This was a cross sectional study involving 141 healthy subjects of either sex, aged between 40 - 75 years. This study was undertaken after the approval by the Institutional Ethical Committee in accordance with the Ethical Standards laid down in the Declaration of Helsinki and obtaining consent from the study participants.

Study protocol: All the test procedures were explained to the study participants before administering the study protocol. Age of all the subjects was noted. In them, the following parameters were quantified: Body mass index, blood pressure (Systolic, diastolic, pulse and mean arterial blood pressure), heart rate, QTC and time domain measures of cardiac vagal function , namely Expiratory: Inspiratory ratio (E:I ratio) and Root mean square of successive $\mathrm{N}-\mathrm{N}$ interval difference ( $\mathrm{r}-\mathrm{MSSD}$ ).

Estimation of body mass index (BMI): The height and weight of all the subjects were measured. BMI was calculated using the formula: weight in kilograms $(\mathrm{kg})$ divided by height in meters $(\mathrm{m})$ squared.

\section{Measurement of blood pressure: Two readings were taken} five minutes apart in the sitting position. The mean of the two was recorded as blood pressure.

Measurement of heart rate (HR): The HR was obtained from counting total number of $R-R$ intervals in one minute electrocardiogram recorded in lead II in supine position in completely relaxed state.

Assessment of QTc: Q - T intervals and the preceding R-R intervals were measured from the electrocardiogram tracing in lead II in supine position. Q -T interval was defined as the first deflection of the QRS complex and the end as the point of maximal change in the slope as the $T$ wave merges with the baseline. QTc was calculated according to Bazzett's formula: QTC = measured QT/square root of the R-R interval. ${ }^{(17)}$

Assessment of time domain measures of cardiac vagal 
function: E:I ratio was quantified during deep breathing and r-MSSD was quantified during normal (spontaneous resting) breathing from lead II electrocardiogram tracing of 60 seconds recorded in supine position.

\section{Procedure followed in deep breathing test and estimation}

of E: I ratio: This test was performed in the morning after subjects were completely relaxed. Before beginning the test, subjects were taught to breathe, at six breaths a minute: five seconds for each inhalation and five seconds for each exhalation. The examiner raised his hand to signal the start of each inhalation and lowered to signal the start of each exhalation. Lead II electrocardiogram was then recorded continuously at a speed of $25 \mathrm{~mm} / \mathrm{s}$ for 60 seconds while the subject breathed as instructed (Cardiart 108T/M K-VII, BPL Ltd. Bangalore, Karnataka, India). The R$R$ intervals were measured accurately from lead II electrocardiogram recorded during deep breathing. The longest $\mathrm{R}-\mathrm{R}$ interval during expiration and the shortest $\mathrm{R}-\mathrm{R}$ interval during inspiration were expressed as Expiratory $\mathrm{E}: \mathrm{I}$ ratio. $^{(13)}$

Recording and Assessment of r-MSSD: r-MSSD was estimated from one minute resting lead II electrocardiogram tracing obtained in supine position in completely relaxed state. All the R-R intervals were measured accurately and fed into a computer. r-M SSD was then estimated with appropriate statistical functions using Microsoft Windows XP Professional (Microsoft Corporation, Redmond, WA, USA).

Stepsfollowed in computing r-M SSD:

Step \#1: The difference between the RR waveform and the delayed waveform was obtained. Step \#2: The differences between the R-R intervals were squared. Step \#3: sum of the squared differences were calculated. Step \#4: The mean of the sum squared differences between the adjacent normal R-R intervals were derived. Step \# 5: The square root of the mean of the sum squared differences between adjacent normal R-R intervals was derived. Step \# 6: The square root of the mean of the sum squared differences between the adjacent normal $R-R$ intervals in the record was divided by the number of $R-R$ intervals within a given time minus one R-Rinterval.

\section{Statistical analysis}

Statistical analysis was performed suitably by employing, Students unpaired t test and Pearson Correlation Coefficient test. The level of significance was determined by two tailed test. Statistical significance was taken to be at p-value less than 0.05 .

\section{Results:}

The results of data analysis are presented below with suitable subheadings

\section{Baseline characteristics of study subjects}

In a total of 141 healthy subjects enrolled into the study 87 were males and 54 were females. The age range was 34 years to 79 years. The mean \pm SD of age, BMI, blood pressure, HR, QTC, E:I ratio and r-M SSD is presented in table 1

\section{E: I ratio and r-MSSD in male and female study subjects}

Data is presented as Mean \pm SD. E: I ratio of males and females did not differ significantly $(1.43 \pm 0.13$ vs. $1.39 \pm$ $0.14, t=1.67, p=0.09$ ). $\quad r-M S S D$ of males did not differ significantly compared to females ( $22.28 \pm 12.64$ vs. 23.94 $\pm 11.34, t=0.778, p=0.431$ )

\section{Correlation between E: I ratio and other measured parameters}

Data on correlation between E: I ratio and other measured parameters is presented in table 3 and fig.1. There was significant negative correlation between $\mathrm{E}: \mathrm{I}$ ratio and age ( $p=0.0027$, Table 2). There was no significant correlation between E:I ratio and body mass index, blood pressure, heart rate and Q-Tc ( Table 2). There was significant positive correlation between E:I ratio and r-MSSD ( $p=0.0064$, fig.1).

\section{Correlation between r-MSSD and other measured parameters}

Data on correlation between r-MSSD and other measured parameters is presented in table 3 . There was significant negative correlation between r-M SSD and age ( $p$ 
$=0.0028$, Table 3). There was significant negative correlation between r-M SSD and systolic blood pressure, diastolic blood pressure, pulse pressure and mean arterial blood pressure $(p=0.012,0.0342,0.0419,0.01$ respectively, Table 3). There was significant negative correlation between r-MSSD and heart rate $(p=0.0001$, Table 3). There was no significant correlation between $r$ M SSD and BMI, blood pressure, HR and QTc (Table 3).

Table 1. The baseline characteristics and cardiac autonomic function parameters in study subjects (values are mean \pm SD)

\begin{tabular}{|l|l|}
\hline Variables & Data $($ Mean \pm SD) \\
\hline Age (years) & $53.12 \pm 9.67$ \\
\hline Body mass index $\left(\mathrm{kg} / \mathrm{m}^{2}\right)$ & $22.50 \pm 3.04$ \\
\hline Systolic blood pressure $(\mathrm{mm} \mathrm{Hg})$ & $122.65 \pm 10.71$ \\
\hline Diastolic blood pressure $(\mathrm{mmHg})$ & $80.80 \pm 4.30$ \\
\hline Pulse pressure $(\mathrm{mmHg})$ & $41.85 \pm 8.62$ \\
\hline M ean arterial blood pressure $(\mathrm{mmHg})$ & $94.75 \pm 5.83$ \\
\hline Heart Rate (beats/minute ) & $79.79 \pm 10.25$ \\
\hline QTC & $0.38 \pm 0.018$ \\
\hline E:I ratio & $1.41 \pm 0.13$ \\
\hline r-MSSD (milliseconds) & $22.92 \pm 12.15$ \\
\hline
\end{tabular}

Table 2. Correlation between E: I ratio and baseline characteristics of healthy subjects

\begin{tabular}{|l|c|c|}
\hline Variables & $\begin{array}{c}\text { Correlation } \\
\text { Coefficient } \\
\text { (r) values }\end{array}$ & p value \\
\hline Age (years) & -0.25 & 0.002 \\
\hline Body mass index $\left(\mathrm{kg} / \mathrm{m}^{2}\right)$ & 0.067 & 0.428 \\
\hline Systolic blood pressure $(\mathrm{mmHg})$ & 0.028 & 0.735 \\
\hline Diastolic blood pressure $(\mathrm{mmHg})$ & -0.030 & 0.722 \\
\hline M ean arterial blood pressure $(\mathrm{mmHg})$ & 0.002 & 0.975 \\
\hline Pulse pressure (mmHg) & 0.050 & 0.550 \\
\hline Resting heart rate (beats/minute) & 0.006 & 0.935 \\
\hline Q-Tc & -0.074 & 0.379 \\
\hline
\end{tabular}

Table 3 Correlation between r-M SSD and other measured parameters in healthy subjects

\begin{tabular}{|l|c|c|}
\hline Variables & $\begin{array}{c}\text { Correlation } \\
\text { Coefficient } \\
\text { (r) values }\end{array}$ & p value \\
\hline Age (years) & -0.25 & 0.002 \\
\hline Body mass index $\left(\mathrm{kg} / \mathrm{m}^{2}\right)$ & -0.039 & 0.63 \\
\hline Systolic blood pressure $(\mathrm{mmHg})$ & -0.21 & 0.01 \\
\hline Diastolic blood pressure $(\mathrm{mmHg})$ & -0.178 & 0.03 \\
\hline M ean arterial blood pressure ( $\mathrm{mmHg})$ & -0.216 & 0.01 \\
\hline Pulse pressure (mmHg) & -0.171 & 0.04 \\
\hline Resting heart rate (Beats/minute) & -0.391 & 0.0001 \\
\hline Q-Tc & 0.089 & 0.291 \\
\hline
\end{tabular}

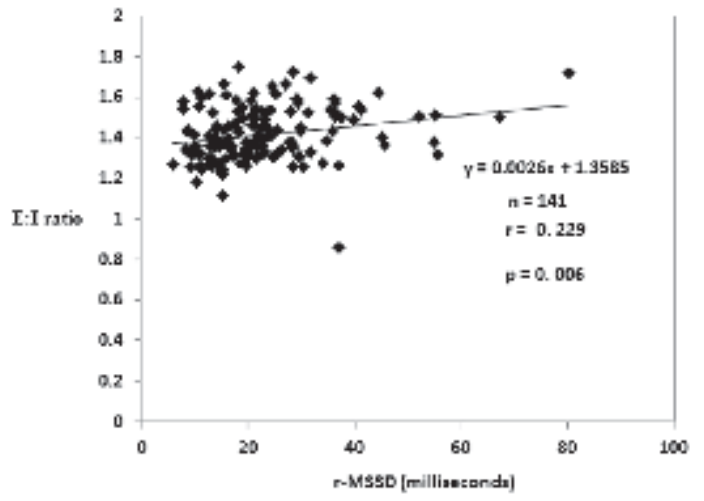

Fig. 1 : Correlation between $r-M S S D$ and $E: I$ ratio in healthy subjects. $Y+0.0026 x+1.3585, n=$ sample size, $r=0.229, p=0.006$, where $\mathrm{y}=\mathrm{E}$ : I ratio and $\mathrm{x}=\mathrm{r}-\mathrm{MSSD}$.

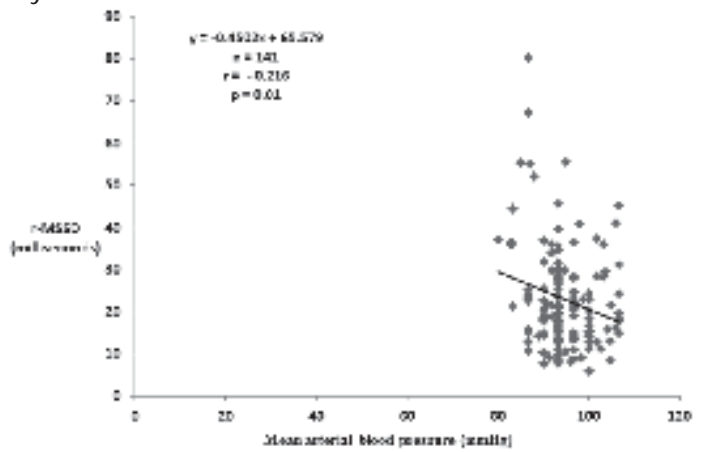

Fig. 2 : Correlation between mean arterial blood pressure and $r$ MSSD in healthy subjects. $Y=-0.4502 x+65.579$, where $y=r$ MSSD and $x=$ mean arterial blood pressure. $n=$ sample size, $r=$ $0.216, p=0.01$

\section{Discussion :}

E:I ratio quantified during deep breathing at 6 respiratory cycles/ minute and r-MSSD obtained during spontaneous breathing at rest in supine position are the two commonly used indices of cardiac parasympathetic function. However, the influencing factors of parasympathetic activity reflected in these two indices are inconclusive. Therefore in this study we compared the characteristics of of E:I ratio and r-MSSD in relation to age, gender, BMI, blood pressure, heart rate and QTc in healthy subjects.

E: I ratio is a widely used indicator of parasympathetic function. ${ }^{(13)} r-M S S D$ is also an indicator of cardiac vagal function. ${ }^{(14 a n d 15)}$ Accordingly in our study significant positive correlation was observed between E: I ratio and r-MSSD (fig. 1). However this significance was at Pearson correlation coefficient 0.229 . This observation suggests that among the several determinants of cardiac parasympathetic activity, only some factors commonly influence $\mathrm{E}$ : I ratio and r-M SSD. 
In the present study, significant negative correlation was observed between age and E: I ratio (Table 2). Significant negative correlation was also observed between r-M SSD and age (Table 3) suggesting vagal control of heart reduces with ageing. The effect of ageing on $\mathrm{HRV}$ is well known. All the studies involving to date have found progressive reduction in the response with increasing age. ${ }^{(18,19,20 \text { and 21) }}$ Thus our data based on141 healthy subjects is in accordance with the findings of previous studies.

In the present study, significant negative correlation was observed between r-M SSD and blood pressure including systolic, diastolic, pulse and mean arterial pressure (Table 3 and Fig. 2). There was no significant correlation between $\mathrm{E}$ : I ratio and blood pressure (Table 2 ). There are important effects of respiration on the heart and circulatory system. In the central nervous system there are interactions between pacemakers and efferent autonomic tone which directly affect heart rate and blood pressure. There are also variations in vagal tone that centrally mediated and related

\section{References:}

1. Ewing DJ, Campbell IW, Clark BF. The natural history of diabetic autonomic neuropathy. Q.J. Med. 1980; 193: 95-108.

2. Malpas SC, Maling TJB. Heart rate variability and cardiac autonomic function in diabetes. Diabetes 1990; 39: 1177-1181.

3. Pipilis A, Flather M, Ormerod O, Sleight P. Heart rate variability in acute myocardial infarction and its association with infarct site and clinical course. Am J Cardiol 1991; 67: 1137-1139.

4. Rothschild M, Rothschild A, Pfeifer M. Temporary decrease in cardiac parasympathetic tone after acute myocardial infarction. Am J Cardiol 1988; 18: 637-639.

5. Casolo G, Balli E, Taddei T, Amuhasi J, Gori C. Decreased spontaneous heart rate variability in congestive heart failure. AmJ Cardiol 1989; 64: 1162-1167.

6. Cohn JN. Abnormalities of peripheral sympathetic nervous system control in congestive heart failure. Circulation 1990; 82: 159-167 (suppl.).

7. Corr PB, Yamada KA, Witkowski F.X. M echanisms controlling cardiac autonomic function and their relation to arrhythmogenesis. In The Heart and Cardiovascular System (eds. Fozzard HA, Haber E, Jenning, RB, Katz AM ). Raven Press, Newyork, 1986: 1343

8. Nakagawa M, Saikawa T, Ito M. Progressive reduction of heart rate variability with eventual sudden death in two patients. Br. Heart J 1994; 71: 87-88

9. Miller IF, Yeates DB, Wong LB. Heart Rate Variability Analysis - Promise and Fulfillment. Business Briefing: Global Health Care - Advanced medical technologies 2004; 1- 4 .

10. Ewing DJ, Clarke B.F. Diagnosis and management of diabetic autonomic neuropathy. Br M ed J 1982; 285: 916-918.

11. Freeman R. Noninvasive evaluation of heart rate variability. In (ed. Low PA) Clinical Autonomic Disorders. Lippincott-Raven, Philadelphia, 1997; 297-307

12. Ewing DJ, Borsey DQ, Bellavere F, Clarke BF. Cardiac autonomic neuropathy in diabetes: comparison of measures of $R-R$ interval variation. Diabetologia.1981;21:18-24.

13. Sundkvist G, Almer LO, Lilja B. Respiratory influence on heart rate in to respiration. These result in decreased vagal activity occurring during inspiration and an increased vagal activity during expiration. ${ }^{(22)}$ Thus it could be said that those components of cardiac parasympathetic activity involved in regulation of blood pressure influences r-MSSD but not E:I ratio in healthy subjects.

In the present study r-MSSD was negatively correlating with heart rate (Table 3) . But no significant correlation was observed between heart rate and E:I ratio (Table 2) . Resting heart rate is generally considered to be under vagal tone. ${ }^{(23)}$ Significant correlation observed only between $\mathrm{r}$ MSSD and heart rate but not E:I ratio and heart rate suggests that, those aspects of vagal activity regulating heart rate is better quantified in r-M SSD but not in E:I ratio.

Thus based on our study findings it could be concluded that E: I ratio and r-M SSD are correlated. Age is an influencing factor of E: I ratio and r-M SSD. Blood pressure and heart rate are the other determinants of r-M SSD but not of E:I ratio in healthysubjects.

diabetes mellitus. British M edical Journal, 1979; 1: 924-925.

14. Kleiger RE, Stein PK, Bosner MS, Rotman JN. Time domain measurements of heart rate variability. Cardiol Clin 1992; 10:487-498.

15. Sztajzel J. Heart rate variability: a noninvasive electrocardiographic method to measure the autonomic nervous system. Swiss M ed Wkly 2004; 134:514-522.

16. Subbalakshmi N.K, Adhikari PMR, Sathyanarayana Rao KN, Jeganathan PS. Deterioration of cardiac autonomic function over a period of one year in relation to cardiovascular and somatic neuropathy complications in type 2 diabetes mellitus. diabetes research and clinical practice 2012; 97 : 313-321.

17. Bazett HC. An analysis of the time relations of electrocardiograms. Heart 1920; 7: 353-370.

18. Ziegler D, Laux G, Dannehl K, Spüler M, M ühlen H, M ayer P, Gries FA. Assessment of cardiovascular autonomic function: age-related normal ranges and reproducibility of spectral analysis, vector analysis, and standard tests of heart rate variation and blood pressure responses. Diabet M ed 1992 9(2):166-175.

19. Hulegar A. Abhishekh, Palgun Nisarga, Ravikiran Kisan, Adoor Meghana, Sajish Chandran, Trichur Raju, Talakad N. Sathyaprabha. Influence of age and gender on autonomic regulation of heart. Journal of Clinical M onitoring and Computing 2013; 27: 259-264.

20. O'Brien IA, O'Hare JP, Lewin IG, Corrall RJ. The prevalence of autonomic neuropathy in insulin-dependent diabetes mellitus: a controlled study based on heart rate variability. QJ M ed 1986; 61:957-967.

21. Smith SA. Reduced sinus arrhythmia in diabetic autonomic neuropathy: diagnostic value of an age-related normal range. $\mathrm{Br} \mathrm{Med}$ J (Clin ResEd) 1982; 285:1599-601.

22. Cowvell. Cardiovascular control and integrated responses. In (ed. West JB) Best and Taylor's Physiological Basis of M edical Practice. Williams and Wilkins, Baltimore/ London. 1984; 263-282.

23. Talman WT, Benaroch EE. Neural control of cardiac function. In (eds. Dyck PJ, Thomas PK, Lambert EH, Bunge R). Peripheral Neuropathy. W.BSaunders, Philadelphia 1992: 177-185. 